\title{
3D DYNAMICAL MODEL FOR LIQUID SLOSHING SIMULATION IN A PARTIALLY FILLED ELLIPTICAL TANK
}

\author{
O. Noui \\ Department of Applied Science \\ University of Quebec at Chicoutimi \\ Chicoutimi, Qc, Canada \\ omar.noui@uqac.ca
}

\author{
M. Bouazara \\ Department of Applied Science \\ University of Quebec at Chicoutimi \\ Chicoutimi, Qc, Canada \\ mbouazar@uqac.ca
}

\author{
M. J. Richard \\ Department of Mechanical Engineering \\ Laval University \\ Quebec, Qc, Canada \\ mrichard@gmc.ulaval.ca
}

\begin{abstract}
Many of 2D mechanical models have been developed to simulate liquid sloshing of a partially filled tank with different shapes. However, those models didn't represent properly the complex liquid motion, especially in the case of portable tanks. Indeed, forces exerted on the liquid can be lateral, longitudinal and vertical. Then, liquid displacement and pressure forces applied to the tank walls are undervalued and may cause design flaws. In this case, 2D mechanical models are ineffective for liquid motion simulation. In previous studies, a $3 \mathrm{D}$ equivalent mechanical model has been developed. This dynamical model is used to simulate different liquid motion in a partially filled tank that consider any sort of excitement forces and get more accurate results in terms of displacements and pressure forces. In this study, a brief description of the new dynamical model is given, including the liquid discretization process, stiffness and damping coefficients computing method and equations of motion. Afterward, the model is applied to an elliptical cross section tank to obtain displacement and pressure forces of the liquid. Finally, the results are compared to the literature.
\end{abstract}

Keywords: liquid sloshing, tank truck, equivalent model, elliptical tank, 3D simulation.

\section{INTRODUCTION}

There are several equivalent mechanical models that simulate 2D liquid movement in a partially filled tank with different shapes such as spring-mass and simple pendulum models [1-4]. The use of such models in the liquid simulation provides convincing results when it comes to lateral sloshing. However, they didn't represent the global liquid motion in a tank. On the other hand, 3D mechanical models as spring-mass lattice are used for modeling complex motions of deformable bodies. Especially in computer graphical animation, springmass systems are often used for their simplicity and rapid implementation [5-7]. Moreover, the use of these models in the simulation of deformable bodies provides both geometrical and physical aspects. In a previous study, a new equivalent 3D dynamical model was developed to simulate the global motion of the liquid in a horizontal tank [8]. A mathematical method for liquid discretization showed that liquid splits in multiple particles along each axis. Then, the masse and coordinates of the center of mass of each particle are computed. The movement of each particle is simulated by the displacement of its center of mass. All centers of mass constitute the nodes of the mesh. Each two adjacent nodes are linked by flexible edges having a parallel spring and damper. To adapt the model to all used forms of tanks, the generic tank cross-section, developed by Kang [9], was employed. In another study [10], we showed how to obtain stiffness and damping coefficient depending on the liquid and the tank design. Afterward, equations of motion were obtained and solved using a computation software program, namely Maple 16 [11]. In this study, the 3D dynamical model is applied to simulate liquid motion and pressure forces in a partially filled tank with an elliptical cross-section. Indeed, this tank shape is one of the most used shapes. Many researches have been dedicated to study this tank geometry. It allows a lower center of mass comparing to a cylindrical tank. We show a brief description of the dynamical model. Afterward, some results are generated and compared to the literature.

\section{3D DYNAMICAL MODEL}

\section{A. Liquid discratization}

In this study, the tank lateral walls are assumed to be straight without any camber. The liquid discretization is made by dividing the length $X$ of the tank by $M$ parts, the height $Z_{h}$ of the free surface by $P$ parts and each portion between two heights $k . Z_{h} / P$ and $(k-1) \cdot Z_{h} / P$ by $N$ parts in each direction of the $\overrightarrow{O Y}$ axis (left side and right side of the $\overrightarrow{O Z}$ axis), with $1 \leq k \leq$ $P$ and $M, N, P \in \mathbb{N}^{*}$. Thus, we obtain $M .2 N$. $P$ particles, noted $p_{i, j, k}$ with $1 \leq i \leq M, 1 \leq j \leq 2 N$ and $1 \leq k \leq P$. Note that the positions of each couple of particles $p_{i, N-(l-1), k}$ and $p_{i,(N+1)+(l-1), k}$ are symmetric with respect to the $\overrightarrow{O Z}$ axis $(1 \leq$ $l \leq N)$. Each particle is represented by its center of mass, noted $G_{i, j, k}$, which are the nodes of the model. Mass $m_{i, j, k}$ and coordinates $\vec{p}_{i, j, k}$ of each node are computed depending on its location [8]. The following figures show an example of the liquid discretization for a half full elliptical tank with $M=12$, $N=4$ and $P=5$ : 


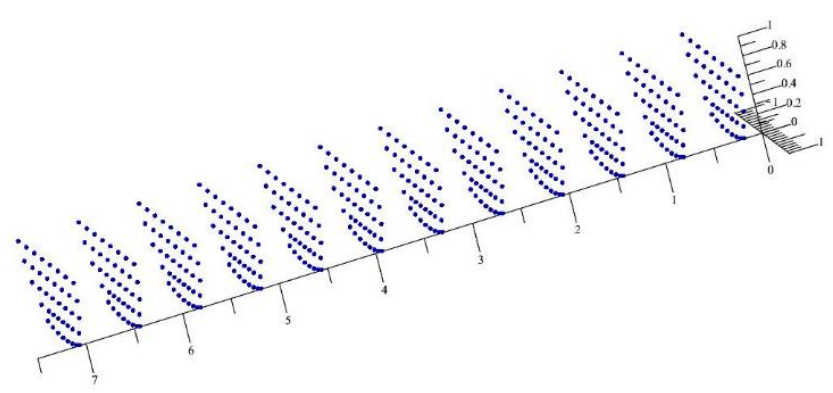

Figure 1: Example of liquid discretization in 3D

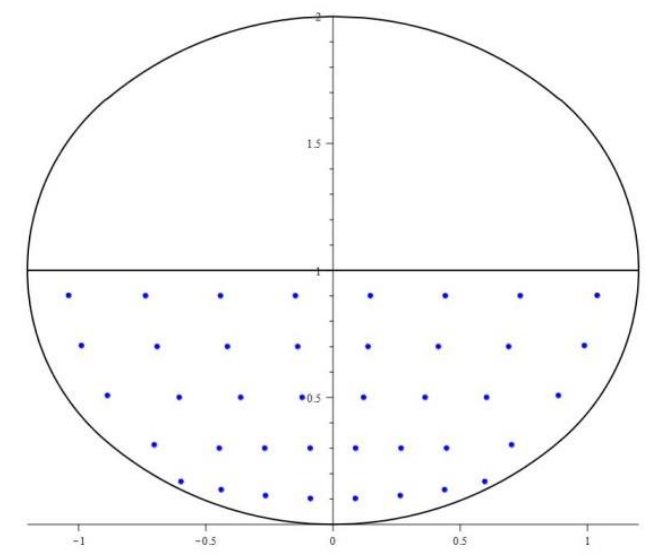

Figure $2: 2 \mathrm{D}$ view of the liquid discretization.

In this example, the model contains of 480 nodes. Then, we will obtain 1440 equations of motion, three equations for each node.

\section{B. Stiffness and damping coefficient}

Each node $G_{i, j, k}$ is linked to six links: two links $A_{1, i, j, k}$ and $A_{1, i+1, j, k}$ along the $\overrightarrow{O X}$ axis, two links $A_{2, i, j, k}$ and $A_{2, i, j+1, k}$ along the $\overrightarrow{O Y}$ axis and two links $A_{3, i, j, k}$ and $A_{3, i, j, k+1}$ along the $\overrightarrow{O Z}$ axis as shown in the following scheme:

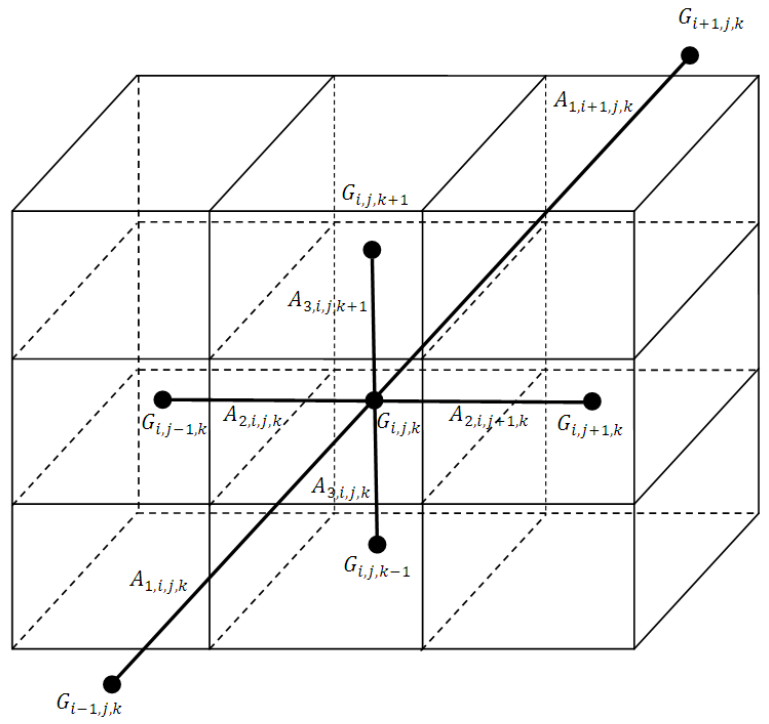

Figure 3: Links between a node $\boldsymbol{G}_{\boldsymbol{i}, \boldsymbol{j}, \boldsymbol{k}}$ and its adjacent nodes.
Each link $A_{q, i, j, k}$, with $q=1,2,3$, contains a parallel linear spring with a stiffness $K_{q, i, j, k}$ and linear damper with a damping coefficient $c_{q, i, j, k}$ as shown in the figure below:

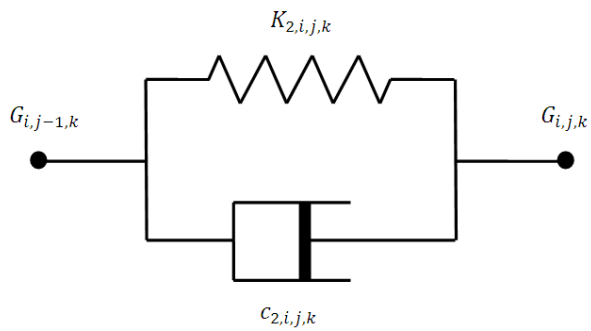

Figure 4: composition of the flexible link.

The nodes contacting the tank walls $G_{1, j, k}, G_{M, j, k}, G_{i, 1, k}$, $G_{i, N, k}$ and $G_{i, j, 1}$ are attached to the structure by the same type of the links. Another special case of the nodes $G_{i, j, P}$, that represent the free surface of the liquid, are linked by only 5 links because they have no contact with the tank structure. The methodology used to compute stiffness and damping coefficient of the model is discussed as follows:

Firstly, stiffness $K_{1, i, j, k}$ along the $\overrightarrow{O X}$ axis is computed. It is supposed that the liquid is divided along the $\overrightarrow{O Z}$ axis. Each part of the liquid between two heights $Z_{k-1}$ and $Z_{k}$ represents a vibration mode. The following formula is used to compute the natural frequency $\omega_{n}$ of each vibration mode $n$ of the liquid in rectangular tank [12]:

$$
\omega_{n}^{2}=\pi(2 n-1) \frac{g}{a} \tanh \left[\pi(2 n-1)\left(\frac{h}{a}\right)\right]
$$

Where $a$ is the width of the tank, $h$ is the height of the liquid and $g=9.81 \mathrm{~m} \cdot \mathrm{s}^{-2}$ is the gravity acceleration. This formula is adapted to our model and becomes:

$$
\omega_{x k}{ }^{2}=\pi(2 n-1) \frac{g}{L} \tanh \left[\pi(2 n-1)\left(\frac{Z_{h}}{L}\right)\right]
$$

Where $n=P-k+1$. Indeed, when the cylinder is placed horizontally, the tank may be considered as rectangular [1]. Then, stiffness $K_{x k}$ of each spring that simulate each mode $k$ is computed by:

$$
K_{x k}=m_{x k} \cdot \omega_{y k}^{2}
$$

Where $m_{x k}$ is the mass of the liquid between two heights $Z_{k-1}$ and $Z_{k}$. Afterward, it is supposed that each spring $K_{x k}$ is a set of $M+1$ springs in series with equal stiffness $K_{x k, i}$ computed by the following formula:

$$
K_{x k, i}=(M+1) \cdot K_{x k}
$$

Then, it is supposed that each spring $K_{x k, i}$ is a set of parallel springs with the same stiffness $K_{1, i, j, k}$ such as:

$$
K_{1, i, j, k}=\frac{K_{x k, i}}{2 N} \text { with } 1 \leq j \leq 2 N
$$

Finally, proceeding by the same way for each part of the liquid between two heights $Z_{k-1}$ and $Z_{k}$, all stiffness along the $\overrightarrow{O X}$ axis are obtained.

Secondly, stiffness $K_{2, i, j, k}$, along the $\overrightarrow{O Y}$, axis are computed. The method is identical to the one used previously for stiffness along the $\overrightarrow{O X}$ axis. It is supposed that the liquid is divided along the $\overrightarrow{O Z}$ axis. The formula (1) that compute natural 
frequencies of each part of the liquid between $Z_{k-1}$ and $Z_{k}$ is adapted as follows:

$$
\omega_{y k}^{2}=\pi(2 n-1) \frac{g}{Y_{k-1}+Y_{k}} \tanh \left[\pi(2 n-1)\left(\frac{Z_{h}}{Y_{k-1}+Y_{k}}\right)\right](6)
$$

Where $Y_{k}$ is the half width of the tank corresponding to the height $Z_{k}$. In fact, the width of the tank along the $\overrightarrow{O Y}$ axis varies depending on the height of the studied liquid part. Therefore, the value of width $a$ in the formula (1) is replaced by the average width between to the heights $Z_{k-1}$ and $Z_{k}$. The stiffness $K_{y k}$ describing each mode is computed by:

$$
K_{y k}=m_{y k} \cdot \omega_{y k}^{2}
$$

Where $m_{y k}$ is the mass of the liquid between $Z_{k-1}$ and $Z_{k}$. It is supposed that the spring $K_{y k}$ is a set of $M$ parallel springs with the same stiffness:

$$
K_{y k, i}=\frac{K_{y k}}{M} \text { with } 1 \leq i \leq M
$$

Afterward, we split each stiffness $K_{y k, i}$ in $2 N+1$ springs in series with equal stiffness, noted $K_{2, i, j, k}$, with:

$$
K_{2, i, j, k}=(2 N+1) \cdot K_{y k, i}
$$

All stiffness $K_{2, i, j, k}$ values along the $\overrightarrow{O Y}$ axis are then obtained by the proposed computational procedures.

Thirdly, the stiffness $K_{3, i, j, k}$ of springs along the $\overrightarrow{O Z}$ axis is computed. In this case, there is no formula in the literature that can be used to compute natural frequencies of vertical sloshing. For this, an important assumption is considered. Indeed, the vertical displacement of the liquid is usually limited. For conventional shapes of the tank, vertical displacement $\Delta z$ of the liquid center of mass is $0.04 \mathrm{~m}$ on average [9]. Considering this assumption, first, it is supposed that there is only one spring, namely $K_{z}$, attached to the center of mass of the liquid. The maximum stretch of this spring should be $\Delta z$. Assuming that the mass of the center of mass is the total liquid mass $m_{t}$, then, stiffness $K_{Z}$ is computed as follows:

$$
K_{z}=\frac{m_{t} \cdot g}{\Delta z}
$$

Then, the spring $K_{z}$ is considered as a set of $P$ springs in series with equal stiffness, noted $K_{z k}$ with $1 \leq k \leq P$. Each stiffness $K_{z k}$ to be calculated by the formula:

$$
K_{z k}=P . K_{z z}
$$

Afterwards, each spring $K_{z k}$ is considered to be a set of $2 M N$ parallel springs $K_{3, i, j, k}$ computed as follows:

$$
K_{3, i, j, k}=K_{z k} / 2 M N
$$

Stiffness of each spring $K_{3, i, j, k}$ along the $\overrightarrow{O Z}$ axis is then obtained.

Finally, the damping coefficients $c_{q, i, j, k}$ with $q=1,2,3$ are assumed to be equal to 100 times the dynamical viscosity coefficient $\eta$ of the liquid multiplied by the corresponding link length. For example, the damping coefficient of a link between two nodes $G_{i, j, k}$ and $G_{i+1, j, k}$ along the $\overrightarrow{O X}$ axis

$$
c_{1, i, j, k}=100 . \eta \cdot\left\|\vec{p}_{i, j, k}-\vec{p}_{i-1, j, k}\right\|
$$

Where $\|\vec{x}\|$ is the Euclidian norm of the vector $\vec{x}$. To obtain consistent results, a calibration factor is added to the model such that all the values of stiffness along $\overrightarrow{O X}$ axis and $\overrightarrow{O Y}$ axis to be multiplied by this factor. The value of this factor is used depending on the number of nodes in the model. Virtually, as the number of nodes decreases extremely, the calibration factor increases. This factor can be explained by the lack of precision when the mesh is less refined. In this case, parameters of the links are underestimated and should be corrected.

\section{Equations of motion}

Displacement, velocity and acceleration vectors of a node $G_{i, j, k}$ are noted respectively $\vec{r}_{i, j, k}(t), \vec{v}_{i, j, k}(t)$ and $\vec{a}_{i, j, k}(t)$. The forces applied on each node are: stress force $\vec{T}_{i, j, k}$ of the springs attached to the nodes, damping force $\vec{A}_{i, j, k}$ and input force $\vec{F}_{i, j, k}$. Stress force $\vec{T}_{i, j, k}$ is calculated by the following formula:

$$
\begin{gathered}
\vec{T}_{i, j, k}=\vec{T}_{1, i, j, k}+\vec{T}_{1, i+1, j, k}+\vec{T}_{2, i, j, k}+\vec{T}_{2, i, j+1, k}+\vec{T}_{3, i, j, k} \\
+\vec{T}_{3, i, j, k+1}
\end{gathered}
$$

Where the stress force of each spring attached to the node is computed by the Hook law. For example:

$$
\begin{aligned}
\vec{T}_{1, i, j, k}=-K_{1, i, j, k} & \left(\left\|\vec{r}_{i, j, k}-\vec{r}_{i-1, j, k}\right\|\right. \\
& \left.-\left\|\vec{p}_{i, j, k}-\vec{p}_{i-1, j, k}\right\|\right) \cdot \frac{\vec{r}_{i, j, k}-\vec{r}_{i-1, j, k}}{\left\|\vec{r}_{i, j, k}-\vec{r}_{i-1, j, k}\right\|}
\end{aligned}
$$

Damping force $\vec{A}_{i, j, k}$ of each node is computed by:

$$
\begin{gathered}
\vec{A}_{i, j, k}=\vec{A}_{1, i, j, k}+\vec{A}_{1, i+1, j, k}+\vec{A}_{2, i, j, k}+\vec{A}_{2, i, j+1, k} \\
+\vec{A}_{3, i, j, k}+\vec{A}_{3, i, j, k+1}
\end{gathered}
$$

Where the damping forces applied by each attached damper is computed by the viscosity law. For example:

$$
\vec{A}_{1, i, j, k}=-c_{1, i, j, k} \cdot\left(\vec{v}_{i, j, k}-\vec{v}_{i-1, j, k}\right)
$$

The input force $\vec{F}_{i, j, k}$ is calculated by the formula below:

$$
\vec{F}_{i, j, k}=m_{i, j, k}\left[\begin{array}{lll}
a_{x} & a_{y} & a_{z}
\end{array}\right]^{t}
$$

Where $a_{x}, a_{y}$ and $a_{z}$ are respectively the longitudinal, lateral and vertical accelerations applied on the tank. Using the Newton second law, we obtain for each node $G_{i, j, k}$ the following equations of motion:

$$
\vec{T}_{i, j, k}+\vec{A}_{i, j, k}+\vec{F}_{i, j, k}=m_{i, j, k} \cdot \vec{a}_{i, j, k}
$$

To obtain the global system of motion equations, the global displacement vector is computed by $\vec{r}$, the global velocity vector by $\vec{v}=\overrightarrow{\dot{r}}$ and the global acceleration vector by $\vec{a}=\overrightarrow{\dot{r}}$. These 3 vectors contain $3 M N P$ components. Moreover, note by $[\mathcal{M}]$ the $3 M N P \times 3 M N P$ diagonal mass matrix such as each mass $m_{i, j, k}$ is repeated 3 times. The global input forces vector is then given by:

$$
\vec{F}=[\mathcal{M}] \cdot \overrightarrow{a_{\text {ext }}}
$$

where $\overrightarrow{a_{\text {ext }}}$ is given by:

$$
\overrightarrow{a_{\text {ext }}}=\left[\begin{array}{lllllll}
a_{x} & a_{y} & a_{z} & \ldots & a_{x} & a_{y} & a_{z}
\end{array}\right]^{t}
$$

The global stress forces $3 M N P$-vector $\vec{T}$ is equal to:

$$
\vec{T}=\left[\begin{array}{lllllll}
T_{x, 1,1,1} & T_{y, 1,1,1} & T_{z, 1,1,1} & \ldots & T_{x, M, N, P} & T_{y, M, N, P} & T_{z, M, N, P}
\end{array}\right]^{t}
$$

Similarly, global damping forces $3 M N P$-vector $\vec{A}$ is obtained by the same manner. Finally, the global system of equations of motion is given as follows:

$$
[\mathcal{M}]\left(\overrightarrow{\ddot{r}}-\overrightarrow{a_{e x t}}\right)+\vec{A}-\vec{T}=0
$$




\section{RESULTS AND DISCUSSION}

In this section, the new $3 \mathrm{D}$ dynamical model is used to simulate liquid sloshing in a partially filled elliptical tank that is one of the most used geometry of portable tanks. The purpose of this simulation is to compare liquid displacement and pressure forces obtained by this model to the results obtained in the literature by two different methods [13]. The first model is an analytical model considering the liquid as a rigid body. Liquid motion is simulated by the movement of center of mass of the body. The second method is obtained by using a numerical simulation software. We compare maximum, both lateral and vertical, displacements of the center of mass of the liquid and maximum pressure forces generated by liquid sloshing.

To obtain lateral displacement $Y_{g}$ and vertical displacement $Z_{g}$ of the center of mass of the liquid using the 3D model developed in this study, the following formulas are used:

$$
\begin{aligned}
& Y_{g}=\frac{1}{m_{T}} \sum_{\substack{i=1, j=1, k=1 \\
M, 2 N, P}}^{M, 2 N, P} m_{i, j, k} \cdot y_{i, j, k} \\
& Z_{g}=\frac{1}{m_{T}} \sum_{i=1, j=1, k=1}^{M, 2 N, P} m_{i, j, k} \cdot z_{i, j, k}
\end{aligned}
$$

Where $m_{T}$ is the total mass of the liquid, $y_{i, j, k}$ and $z_{i, j, k}$ are respectively lateral and vertical displacement of the node $G_{i, j, k}$. Using the forth order numerical derivation, lateral acceleration is computed to obtain lateral pressure force $F_{y}$.

A $50 \%$ filled elliptical tank is considered in this simulation with $X=7.5 \mathrm{~m}, a=1.2 \mathrm{~m}$ and $b=1 \mathrm{~m}$. The liquid is supposed to be a domestic oil with a density $\rho=966 \mathrm{~kg} \cdot \mathrm{m}^{-3}$ and a dynamic viscosity $\eta=0.048 \mathrm{~kg} \cdot \mathrm{m}^{-1} \cdot \mathrm{s}^{-1}$. The selected values for the discretizing model are $M=12, N=4$ and $P=$ 5 . Therefore, the dynamical model consists of 480 nodes and 585 links (Figure 1). Firstly, a vehicle engagement in a curve maneuver is used as an input force with maximum lateral acceleration $A=3 \mathrm{~m} \cdot \mathrm{s}^{-2}$. The following table shows maximum displacements and lateral pressure forces obtained by three different models.

TABLE I. COMPARING RESULTS OF LIQUID DISPLACEMENTS AND PRESSURE FORCES ACCORDING TO THE NEW 3D MODEL AND THE LITERATURE.

\begin{tabular}{|c|c|c|c|}
\cline { 2 - 3 } \multicolumn{1}{c|}{} & \multicolumn{2}{c|}{ Literature } & \multirow{2}{*}{ New 3D } \\
\cline { 2 - 3 } \multicolumn{1}{c|}{} & $\begin{array}{c}\text { Analytical } \\
\text { Model }\end{array}$ & $\begin{array}{c}\text { Numerical } \\
\text { Model }\end{array}$ & \\
\hline Lateral displacement $(m)$ & 0.18 & 0.27 & 0.246 \\
\hline Vertical displacement $(m)$ & 0.023 & 0.073 & 0.111 \\
\hline Lateral pressure force $(\mathrm{KN})$ & 40.5 & 52 & 51.05 \\
\hline
\end{tabular}

The table above shows accurate results in term of displacements and lateral pressure forces. Indeed, the numerical model using the simulation software is supposed to be the most accurate with respect to real displacements and lateral pressure forces. Note that the results obtained by the new 3D model are closer to the numerical model than the analytical model of the literature [13]. We can also notice that the lateral pressure forces are undervalued by the analytical model while the new 3D model developed in this study is more accurate. Thus, these results validate the new $3 \mathrm{D}$ model and it is possible to use it for tank design and for the study of vehicle stability. Note also that for the simulation of the center of mass motion for a duration of 12 seconds, the computer required approximately one hour of computation time. Which is much shorter than the computation time of a numerical simulation software which requires hours, or even days of computation. Then we can show the real results obtained by the new model by evaluating displacement of each node of the mesh. The figure below shows the displacement of the liquid after one second of motion in a curve:

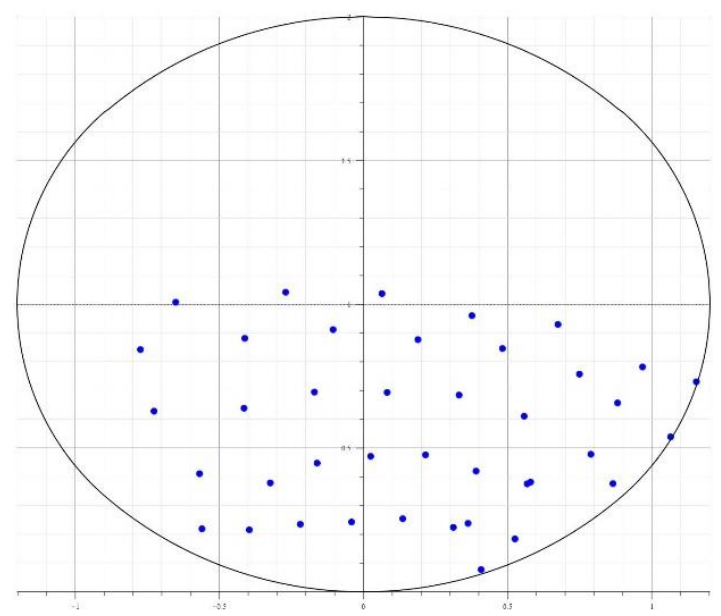

Figure 5: liquid displacement after $1 \mathrm{~s}$ of the vehicle engagement in a curve.

Figure 6 shows displacement of each node representing the liquid after two seconds of the vehicle movement in a curve:

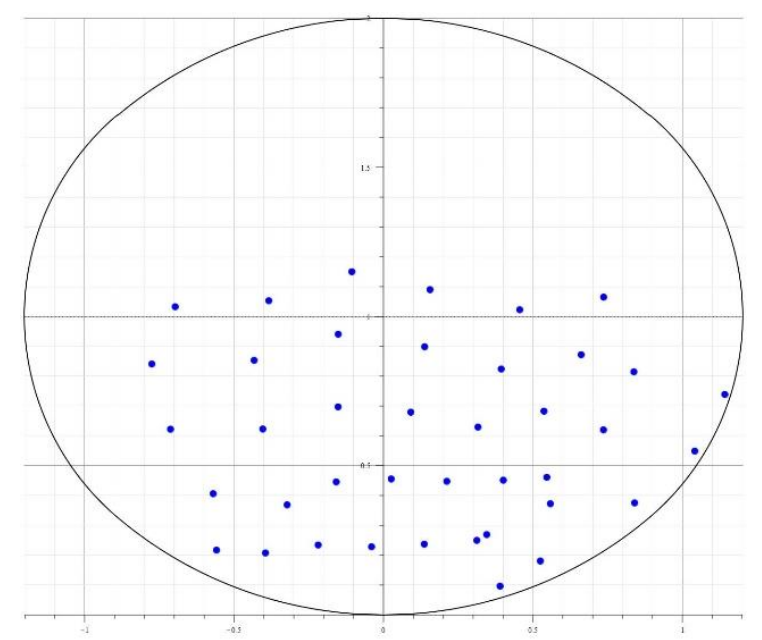

Figure 6: liquid displacement after $2 \mathrm{~s}$ of the vehicle engagement in a curve.

The two figures above show that not only does the new model provide accurate values of displacement of the center of mass and pressure forces generated by the liquid motion, but it is also possible to visualize the global liquid motion by evaluating displacement of each node. In addition, it is also possible to find an approximation of the shape of the liquid free surface by linking the adjacent nodes that represent the free surface. 


\section{CONCLUSION}

In this study, a new 3D-dynamical model is described and used to simulate liquid sloshing in an elliptical tank. Firstly, we showed a brief description of the dynamical model. This model is based on the idea of modeling the liquid as a deformable body using a spring-mass lattice. A special method is used for liquid discretization. Each mass of the liquid is simulated by a node of the mesh that represents its center of mass. The adjacent nodes are linked by a flexible link containing a parallel spring and damper. Afterwards, stiffness and damping coefficient of each link are computed using some assumptions. The equations of motion of each node are then calculated and compiled to obtain the global system of equations of the 3D model. All formulas and equations are programmed and solved using Maple 16 software. To compare the results of the model with the literature, identical dimensions of an elliptical tank are used as well as the filling rate, the external force applied and the liquid properties. Comparing the results with the literature allows to conclude that the new model provide results closer to the numerical models that require a longer computation time. Moreover, the new model allows a visualization of the global movement of the liquid by evaluating displacement of each node of the mesh. Future works will focus on generating more results for other tank geometries, filling rates and other vehicle maneuver. It will be possible to compare results generated by this new model with other researches.

\section{ACKNOWLEDGMENT}

The work reported in this article is financed by the natural sciences and engineering research council of Canada (NSERC) and the university of Quebec at Chicoutimi.

\section{REFERENCES}

[1] H. N. Abramson (1966), «The Dynamic Behavior of Liquids in Moving Containers». NASA SP-106.

[2] R. Ranganathan and al (1993), «Analysis of fluid in partially filled tanks and their impact on the directional response of tank vehicles». SAE Technical Paper 932942, p. 39-45.

[3] A. A. El Damatty, A.M.I. Sweedan (2006), « Equivalent mechanical analog for dynamic analysis of pure conical tanks », Thin-Walled Structures 44(2006) 429-440.

[4] O. Noui (2012), "Étude et modélisation des citernes en aluminium pour véhicule routier ", Master's thesis, University Of Quebec at Chicoutimi.

[5] X. Provot (1995), « Deformation constraints in a Mass-Spring Model to Describe Rigid Cloth Behavior », National Institute for Research in Computer Science and Control (France).

[6] M. Balci, H. Foroosh (2006), « Real-time 3D fire simulation using a springmass model ». Multi-Media modelling conference proceedings, 2006 12th International, Beijing, 10.1109/MMMC.2006.1651309

[7] J. Mesit, R. Guha, S.Chaudhry (2007), « 3D soft Body Simulation Using Mass-Spring System with Internal Pressure Force and Simplified Implicit Integration », Journal Of Computers, Vol. 2, No. 8.

[8] M. Bouazara, O. Noui and M. J. Richard (2014), «3D mechanical model for liquid motion simulation in a partially filled tank $», 15^{\text {th }}$ International Mechanical Engineering Congress \& Exposition IMECE2014, Palais des congres, Montreal.
[9] X. Kang (2001), «Optimal tank design and directional dynamic analysis of liquid cargo vehicles under steering and braking». Ph.D. Thesis, Concordia University Canada, Montreal. p. 323

[10] O. Noui, M. Bouazara, and M. J. Richard (2016), « Study of liquid motion and pressure forces applied on the walls of partially filled moving tank», $16^{\text {th }}$ International Mechanical Engineering Congress \& Exposition IMECE2016, Phoenix, Arizona, USA.

[11] Maple 16, www.maplesoft.com. MapleSoft, Waterloo, ON, Canada.

[12] F.T. Dodge (2008), " The new dynamic behaviour of liquids in moving containers ». Southwest Research Institute (SRI), San Antonio, Texas (USA).

[13] M. Toumi, M. Bouazara, M. J. Richard (2009), «Impact of liquid sloshing on the behaviour of vehicles carrying liquid cargo», European Journal of Mechanics A/Solids 28 1026-1034. 